41th "Jaszowiec" International School and Conference on the Physics of Semiconductors, Krynica-Zdrój 2012

\title{
Spin Hall Effect in a Two-Dimensional Electron Gas with Strong Rashba Spin-Orbit Interaction: Semiclassical Keldysh Approach
}

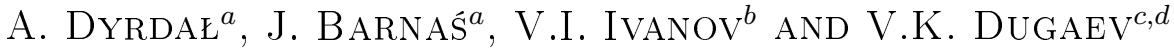 \\ ${ }^{a}$ Faculty of Physics, Adam Mickiewicz University, Umultowska 85, 61-614 Poznań, Poland \\ ${ }^{b}$ Frantsevich Institute for Problems of Materials Science, NASU, Vilde 5, 58001 Chernovtsy, Ukraine \\ ${ }^{c}$ Department of Physics, Rzeszów University of Technology, Powstańców Warszawy 6, 35-959 Rzeszów, Poland \\ ${ }^{d}$ Institut für Physik, Martin-Luther-Universität Halle-Wittenberg, 06120 Halle, Germany \\ Spin Hall effect in a two-dimensional electron gas with the Rashba spin-orbit interaction is analyzed \\ theoretically. We use the Keldysh technique for nonequilibrium processes, modified in order to take into account \\ well-defined splitting of the Fermi surface due to strong spin-orbit coupling. Using such an approach, we \\ reconsider the two-dimensional electron gas with the Rashba spin-orbit interaction and show that impurity \\ scattering processes suppress the spin Hall effect.
}

PACS: 73.63.-b, 72.25.Dc, 75.70.Tj

\section{Introduction}

Spin Hall effect (SHE) is a phenomenon, in which an electric field generates a spin current flowing perpendicularly to the field. The effect has been studied in various systems, but of particular interest are systems where spin current is not accompanied by a charge current. This can happen in nonmagnetic systems with strong spin-orbit (SO) interaction. An external electric field (current in a conducting case) generates then a pure spin current perpendicular to the field. The importance of SHE is due to the fact that it generates pure spin current required for electrical manipulation of magnetic moments.

The SHE has been studied with various techniques, including the Kubo formalism for the off-diagonal conductivity [1-3]. This method allows to calculate the SHE in linear to electric field approximation. As a diagrammatic method, it requires summation of many diagrams, especially in the cases of heavy doping, strong scattering from impurities, or rather smooth (not point-like) impurity potential. All of these problems can be efficiently overcome by using the Keldysh formalism [4, 5]. However, the Keldysh technique in its original form leads to very cumbersome equations when applied to the SHE, which obviously contain excess information [6].

Several mechanisms of the SHE have been proposed so far. One of them is the intrinsic SHE in a clean system. It was studied, e.g., in the model of two-dimensional (2D) electron gas with the Rashba SO interaction. In this model, the spin Hall conductivity $\sigma_{\mathrm{H}}$ of a clean system is equal to a universal constant, but impurities with point-like scattering potential suppress $\sigma_{\mathrm{H}}$ down to zero. This suppression is associated with the vertex correction to the loop diagram [7-9], usually calculated in the ladder approximation [2]. The later is well justified for a simple one-band model in the limit of $E_{\mathrm{F}} \tau \gg 1$, while its validity in a general case of complex band structure is rather not clear.
In this paper we use the Keldysh formalism [4, 5], modified to make it closer to the kinetic equation for the Wigner density matrix. It involves integrated Keldysh functions, similar to the semiclassical approximation [10]. Recently, some modifications of this technique have been used to study SHE in 2D electron gas [11-13]. Our approach is somewhat close, but essentially differs from them by the way how we determine the integrated Keldysh functions. Hence, our approach is a generalization to the case of well-defined splitting of the Fermi surface (strong SO coupling, weak impurity scattering), like in the case of magnetized electron gas. For 2D electron gas with SO interaction, the approach allows considering the regime of strong SO coupling. We found that impurities generally suppress the SHE, in agreement with results obtained earlier by other techniques. Whether the suppression is complete or not, depends on specific intraand inter-band relaxation rates. The latter, in turn, depend on the form of scattering potential.

\section{Model and method}

We consider $2 \mathrm{D}$ electron gas with the Rashba SO interaction. The latter is due to a substrate and has the lattice periodicity. Impurities, in turn, are treated as a small perturbation. Hamiltonian of the system is

$$
H=H_{0}+V(\boldsymbol{r}),
$$

where $H_{0}$ includes the kinetic energy and the Rashba interaction, $H_{0}=-(\Delta / 2 m)-\mathrm{i} \alpha\left(\sigma_{x} \nabla_{y}-\sigma_{y} \nabla_{x}\right)$, while $V(\boldsymbol{r})=\sum_{i} v\left(\boldsymbol{r}-\boldsymbol{R}_{i}\right)$ describes scattering potential due to impurities. Here $\Delta=\partial^{2} / \partial x^{2}+\partial^{2} / \partial y^{2}, \boldsymbol{\sigma}=\left(\sigma_{x}, \sigma_{y}, \sigma_{z}\right)$ are the Pauli spin matrices, whereas $\alpha$ is the constant of the Rashba SO interaction, and we use the units with $\hbar=1$. The energy spectrum of the system without impurities consists of two bands, $E_{1,2}(k)=\varepsilon_{k} \pm \alpha k$, where $\varepsilon_{k}=k^{2} / 2 m$.

The formalism used to calculate SHE is based on nonequilibrium Green functions, which allow to find kinetic equations for the spin-dependent distribution func- 
tions in the semiclassical approximation. We use the matrix Green functions introduced by Keldysh for nonequilibrium processes $[4,5]$, which include the retarded, $G^{\mathrm{R}}$, advanced, $G^{\mathrm{A}}$, and off-diagonal (Keldysh), $G^{\mathrm{K}}$, functions. Each of the three Green functions $\left(G^{\mathrm{R}}, G^{\mathrm{A}}\right.$, and $G^{\mathrm{K}}$ ) is additionally a matrix in the spin space. We also assume that the impurity scattering is weak, so the Born approximation can be used.

In the semiclassical approximation, Green function calculation is based on the gradient expansion. Upon writing the equation of motion for the Green function, performing the Fourier transformation with respect to the time and space variables, and making use of the MahanHansch [1] substitution, one obtains the following equation for the off-diagonal (Keldysh) component of the Green function (valid for a homogeneous in space and time problem):

$$
\begin{aligned}
& \left(e \boldsymbol{E} \cdot \nabla_{\boldsymbol{k}}+\frac{e}{m} \boldsymbol{E} \cdot \boldsymbol{k} \frac{\partial}{\partial \varepsilon}\right) G_{\varepsilon \boldsymbol{k}}^{\mathrm{K}} \\
& \quad+\mathrm{i} \alpha k_{y}\left[\sigma_{x}, G_{\varepsilon \boldsymbol{k}}^{\mathrm{K}}\right]_{-}-\mathrm{i} \alpha k_{x}\left[\sigma_{y}, G_{\varepsilon \boldsymbol{k}}^{\mathrm{K}}\right]_{-} \\
& \quad-\frac{\alpha}{2} e E_{x}\left[\sigma_{y}, \frac{\partial G_{\varepsilon \boldsymbol{k}}^{\mathrm{K}}}{\partial \varepsilon}\right]_{+}+\frac{\alpha}{2} e E_{y}\left[\sigma_{x}, \frac{\partial G_{\varepsilon \boldsymbol{k}}^{\mathrm{K}}}{\partial \varepsilon}\right]_{+} \\
& \quad=-\mathrm{i}\left(\sum_{\varepsilon \boldsymbol{k}}^{\mathrm{R}} G_{\varepsilon \boldsymbol{k}}^{\mathrm{K}}+\Sigma_{\varepsilon \boldsymbol{k}}^{\mathrm{K}} G_{\varepsilon \boldsymbol{k}}^{\mathrm{A}}-G_{\varepsilon \boldsymbol{k}}^{\mathrm{R}} \Sigma_{\varepsilon \boldsymbol{k}}^{\mathrm{K}}-G_{\varepsilon \boldsymbol{k}}^{\mathrm{K}} \Sigma_{\varepsilon \boldsymbol{k}}^{\mathrm{A}}\right)
\end{aligned}
$$

where $\boldsymbol{E}$ denotes the external electric field, $\boldsymbol{k}$ is the 2D wave vector, and the self-energy $\Sigma_{\varepsilon \boldsymbol{k}}$ related to scattering from impurities has the form

$$
\Sigma_{\varepsilon \boldsymbol{k}}=N_{\mathrm{imp}} \int \frac{\mathrm{d}^{2} \boldsymbol{k}^{\prime}}{(2 \pi)^{2}} v_{\mathbf{k k}^{\prime}} G_{\varepsilon \boldsymbol{k}^{\prime}} v_{\boldsymbol{k}^{\prime} \boldsymbol{k}}^{*},
$$

with $N_{\text {imp }}$ denoting the impurity density, and $v_{\mathbf{k k}^{\prime}}$ being the matrix elements of the impurity potential. Equation (2) is the semiclassical kinetic equation for the Keldysh function for a 2D electron system with the Rashba SO interaction. It keeps this form also when we take into consideration scattering by phonons or any other relaxation processes included in the self energy part $\Sigma_{\varepsilon \boldsymbol{k}}$.

Here we restrict our considerations to small deviations from equilibrium, $G_{\varepsilon \boldsymbol{k}} \simeq G_{\varepsilon \boldsymbol{k}}^{0}+\delta G_{\varepsilon \boldsymbol{k}}$. Assuming low impurity concentration, the equilibrium functions $G_{\varepsilon \boldsymbol{k}}^{\mathrm{oR}}$ and $G_{\varepsilon \boldsymbol{k}}^{0 \mathrm{~A}}$ can be written as

$$
G_{\varepsilon \boldsymbol{k}}^{\mathrm{OR}(\mathrm{A})} \approx \frac{\varepsilon-\varepsilon_{k}+\alpha\left(k_{y} \sigma_{x}-k_{x} \sigma_{y}\right)}{\left(\varepsilon-E_{1 k} \pm \mathrm{i} \delta_{1}\right)\left(\varepsilon-E_{2 k} \pm \mathrm{i} \delta_{2}\right)},
$$

where $\delta_{1}$ and $\delta_{2}$ are small numbers describing decoherence of the states formed by the Rashba interaction. The equilibrium Keldysh function, $G_{\varepsilon \boldsymbol{k}}^{0 \mathrm{~K}}$, is then determined by the Fermi distribution function $f(\varepsilon)$ as $[4,5]$ :

$$
G_{\varepsilon \boldsymbol{k}}^{0 \mathrm{~K}}=[1-2 f(\varepsilon)]\left(G_{\varepsilon \boldsymbol{k}}^{0 \mathrm{R}}-G_{\varepsilon \boldsymbol{k}}^{0 \mathrm{~A}}\right) .
$$

Neglecting any energy shift of electron bands by impurities, one may consider only imaginary part of the Green function,

$$
\begin{aligned}
& G_{\varepsilon \boldsymbol{k}}^{0 \mathrm{R}} \simeq-\frac{\mathrm{i} \pi}{2}\left[\delta\left(\varepsilon-E_{1 k}\right)\left(1+\boldsymbol{n}_{\boldsymbol{k}} \cdot \boldsymbol{\sigma}\right)\right. \\
& \left.\quad+\delta\left(\varepsilon-E_{2 k}\right)\left(1-\boldsymbol{n}_{\boldsymbol{k}} \cdot \boldsymbol{\sigma}\right)\right],
\end{aligned}
$$

where the unit vector $\boldsymbol{n}_{\boldsymbol{k}}$ has been introduced

$$
\boldsymbol{n}_{\boldsymbol{k}}=\left(\frac{\alpha k_{y}}{\lambda_{k}},-\frac{\alpha k_{x}}{\lambda_{k}}, 0\right),
$$

and $\lambda_{k}=\alpha k$.

Since we look for small deviations from equilibrium, we may write

$$
G_{\varepsilon \boldsymbol{k}}^{\mathrm{K}} \simeq G_{\varepsilon \boldsymbol{k}}^{0 \mathrm{~K}}+\delta G_{\varepsilon \boldsymbol{k}}^{\mathrm{K}} .
$$

Then, the equation for $\delta G_{\varepsilon \boldsymbol{k}}^{\mathrm{K}}$ takes the form

$$
\begin{gathered}
\boldsymbol{E} \cdot\left(e \nabla_{\boldsymbol{k}}+\frac{e}{m} \boldsymbol{k} \frac{\partial}{\partial \varepsilon}\right) G_{\varepsilon \boldsymbol{k}}^{0 \mathrm{~K}}-\mathrm{i} \alpha k_{x}\left[\sigma_{y}, \delta G_{\varepsilon \boldsymbol{k}}^{\mathrm{K}}\right] \\
+\mathrm{i} \alpha k_{y}\left[\sigma_{x}, \delta G_{\varepsilon \boldsymbol{k}}^{\mathrm{K}}\right]+\frac{\alpha}{2} e E_{y}\left\{\sigma_{x}, \frac{\partial G_{\varepsilon \boldsymbol{k}}^{0 \mathrm{~K}}}{\partial \varepsilon}\right\} \\
-\frac{\alpha}{2} e E_{x}\left\{\sigma_{y}, \frac{\partial G_{\varepsilon \boldsymbol{k}}^{0 \mathrm{~K}}}{\partial \varepsilon}\right\}=-\mathrm{i} \Sigma_{\varepsilon \boldsymbol{k}}^{0 \mathrm{R}} \delta G_{\varepsilon \boldsymbol{k}}^{\mathrm{K}} \\
\quad-\mathrm{i} \delta \Sigma_{\varepsilon \boldsymbol{k}}^{\mathrm{K}} G_{\varepsilon \boldsymbol{k}}^{0 \mathrm{~A}}+\mathrm{i} G_{\varepsilon \boldsymbol{k}}^{0 \mathrm{R}} \delta \Sigma_{\varepsilon \boldsymbol{k}}^{\mathrm{K}}+\mathrm{i} \delta G_{\varepsilon \boldsymbol{k}}^{\mathrm{K}} \Sigma_{\varepsilon \boldsymbol{k}}^{0 \mathrm{~A}},
\end{gathered}
$$

where $\Sigma_{\varepsilon \boldsymbol{k}}^{0}$ is the equilibrium self-energy. We look for a solution of Eq. (9) in the form

$$
\delta G_{\varepsilon \boldsymbol{k}}^{\mathrm{K}}=-\mathrm{i} \pi\left[Q_{\boldsymbol{k}} \delta\left(\varepsilon-E_{1 k}\right)+R_{\boldsymbol{k}} \delta\left(\varepsilon-E_{2 k}\right)\right],
$$
with

$$
Q_{\boldsymbol{k}}=Q_{\boldsymbol{k} 0}+\boldsymbol{Q}_{\boldsymbol{k}} \cdot \boldsymbol{\sigma}, \quad R_{\boldsymbol{k}}=R_{\boldsymbol{k} 0}+\boldsymbol{R}_{\boldsymbol{k}} \cdot \boldsymbol{\sigma} .
$$

Let us note that $Q_{k}$ and $R_{k}$ are defined on the surfaces $\varepsilon=E_{1 k}$ and $\varepsilon=E_{2 k}$, respectively, and depend only on the direction of the vector $\boldsymbol{k}$. We assume now electric field along the $y$ axis, $\boldsymbol{E}=(0, E, 0)$. Furthermore, we simplify the problem by considering spin-independent scattering from impurities, i.e., we take $v_{\mathbf{k k}^{\prime}}$ proportional to the unit matrix in spin space. Taking all this into account we write Eq. (9) in an explicit form and then integrate it over $\varepsilon$. As a result one obtains eight coupled equations for the components of $\tilde{Q}_{\boldsymbol{k}}=\left(Q_{\boldsymbol{k} 0}, Q_{\boldsymbol{k} x}, Q_{\boldsymbol{k} y}, Q_{\boldsymbol{k} z}\right)^{\mathrm{T}}$ and $\tilde{R}_{\boldsymbol{k}}=\left(R_{\boldsymbol{k} 0}, R_{\boldsymbol{k} x}, R_{\boldsymbol{k} y}, R_{\boldsymbol{k} z}\right)^{\mathrm{T}}$. These equations can be solved taking all averages $\left\langle Q_{k \mu}\right\rangle$ and $\left\langle R_{k \mu}\right\rangle(\mu=0, x, y, z)$ over wavevector orientations as known functions. Thus, the set of equations for $Q_{\boldsymbol{k} \mu}$ can be written as

$$
\mathcal{M}_{1 k} \tilde{Q}_{k}=A_{1 k} \text {. }
$$

Here, $\mathcal{M}_{1 \boldsymbol{k}}$ is a $4 \times 4$ matrix,

$$
\mathcal{M}_{1 \boldsymbol{k}}=\left(\begin{array}{cccc}
1 / \tau_{1} & 0 & 0 & 0 \\
0 & 1 / \tau_{1} & 0 & 2 \alpha k_{x} \\
0 & 0 & 1 / \tau_{1} & 2 \alpha k_{y} \\
0 & -2 \alpha k_{x} & -2 \alpha k_{y} & 1 / \tau_{1}
\end{array}\right),
$$

where $1 / \tau_{1}=1 / \tau_{11}+1 / \tau_{12}$, and the components $A_{1 \boldsymbol{k} \mu}$ $(\mu=0, x, y, z)$ of $A_{1 k}$ are defined as:

$$
\begin{gathered}
A_{1 \boldsymbol{k} 0}=e E \frac{\partial \tilde{f}_{1}}{\partial E_{1 k}} \frac{\partial E_{1 k}}{\partial k_{y}}+\frac{\left\langle Q_{k 0}\right\rangle}{\tau_{11}}+\frac{\boldsymbol{n}_{\boldsymbol{k}} \cdot\left\langle\boldsymbol{Q}_{k}\right\rangle}{\tau_{11}} \\
+\frac{\left\langle R_{k^{\prime} 0}\right\rangle}{\tau_{12}}+\frac{\boldsymbol{n}_{\boldsymbol{k}} \cdot\left\langle\boldsymbol{R}_{k^{\prime}}\right\rangle}{\tau_{12}}
\end{gathered}
$$

and

$$
\begin{aligned}
& A_{1 \boldsymbol{k} \mu}=e E \tilde{f}_{1} \frac{\partial n_{\boldsymbol{k} \mu}}{\partial k_{y}}+e E \frac{\partial \tilde{f}_{1}}{\partial E_{1 k}} \frac{\partial E_{1 k}}{\partial k_{y}} n_{\boldsymbol{k} \mu} \\
& +\frac{\left\langle Q_{k \mu}\right\rangle}{\tau_{11}}+\frac{\left\langle R_{k^{\prime} \mu}\right\rangle}{\tau_{12}}+\frac{n_{\boldsymbol{k} \mu}\left\langle Q_{k 0}\right\rangle}{\tau_{11}}+\frac{n_{\boldsymbol{k} \mu}\left\langle R_{k^{\prime} 0}\right\rangle}{\tau_{12}}
\end{aligned}
$$

for $\mu=x, y, z$, where $\tilde{f}_{1 k} \equiv 2 f\left(E_{1 k}\right)-1$, and $k^{\prime}$ obeys 
the condition $E_{2 k^{\prime}}=E_{1 k}$. Thus, $k^{\prime}$ can be considered as a function of $k$.

Similar equations can be written for the components $R_{\boldsymbol{k}^{\prime} \mu}$. Apart from this, in Eq. (13) we have introduced the inter- and intra-band relaxation times, $\tau_{i j}$ for $i, j=1,2$, which are determined by the matrix elements of the scattering potential, $\left|v_{\boldsymbol{k} q}\right|^{2}$, where $\boldsymbol{k}$ and $\boldsymbol{q}$ can belong to the same band or to different bands, and also by the density of states in the two bands, $\nu_{1,2}=(m / 2 \pi)\left|1 \pm m \alpha^{2} / \lambda_{k_{1,2}}\right|^{-1}$.

Upon solving Eqs. (12) and averaging over direction of vector $\boldsymbol{k}$ (and $\boldsymbol{k}^{\prime}$ ) we arrive at a system of 8 linear equations for the average components $\left\langle Q_{k \mu}\right\rangle$ and $\left\langle R_{k^{\prime} \mu}\right\rangle$. Then, substituting the average values back to the equations for the unaveraged components of $\tilde{Q}_{k}$ and $\tilde{R}_{k}$ one obtains a complete solution to the problem.

\section{Spin Hall effect}

Having the distribution function $\delta G_{\varepsilon \boldsymbol{k}}^{\mathrm{K}}$, one can calculate the spin current flowing along the axis $x$,

$$
j_{x}^{s_{z}}=-\frac{1}{2} \int \frac{\mathrm{d}^{2} \boldsymbol{k}}{(2 \pi)^{2}} \frac{k_{x}}{m}\left(Q_{\boldsymbol{k} z}+R_{\boldsymbol{k} z}\right) .
$$

For simplicity we assume that there are no interband scattering transitions $\left(1 / \tau_{12}=1 / \tau_{21}=0\right)$ and $\tau_{11}=$ $\tau_{22}=\tau$. The spin current can be then written in a simple form as

$$
j_{x}^{s_{z}}=\frac{e E}{8 \pi^{2} m^{2}} \int \mathrm{d} k k \mathcal{A}(k),
$$

where

$$
\begin{aligned}
& \mathcal{A}(k)=\left(1+\frac{k}{m \alpha}\right) \frac{\partial \tilde{f}_{1}}{\partial E_{1 k}}+\left(1-\frac{k}{m \alpha}\right) \frac{\partial \tilde{f}_{2}}{\partial E_{2 k}} \\
& +\frac{1}{\alpha k}\left(\tilde{f}_{1}-\tilde{f}_{2}\right) .
\end{aligned}
$$

Performing now the integration over $k$ in Eq. (17) one obtains $j_{x}^{s_{z}}=0$. This means that the SHE is completely suppressed by scattering from impurities, even when the impurity density and/or impurity scattering potential are small. This is in agreement with results obtained within different methods by Inoue et al. [8] and Dimitrova [14] for short-range impurity potential. However, such a complete suppression of SHE appears only for the assumed relation between the relaxation times. For example, when assuming other relations between the inter- and intra-band relaxation times, the suppression is not complete and some spin current survives, leading to a non-zero spin Hall conductivity. It is worth noting that even for defects with point-like (short-range) scattering potentials, the relaxation times are different due to different densities of states in the two bands for strong Rashba coupling. Moreover, the relaxation times depend on the impurity potential range, i.e. on $\left|v_{\boldsymbol{k} \boldsymbol{q}}\right|^{2}$. Assuming particular relaxation times one essentially restricts impurity potentials to some specific shapes.

\section{Conclusion}

We have analyzed $2 \mathrm{D}$ electron gas with the Rashba SO interaction and potential scattering from impurities. We used the Keldysh formalism, which has been modified to take into account two well separated Fermi surfaces. Our considerations were limited to small deviation from equilibrium and also to small density of impurities. The main result of our calculations is the suppression of SHE by scattering from impurities. In general, the SHE induced by the Rashba interaction is reduced by scattering on impurities, even in the limit of low impurity concentration. Perfect suppression appears only for some specific relations between inter- and intra-band relaxation times.

\section{Acknowledgments}

This work is supported by EU under European Social Fund - Operational Programme Human Capital POKL.04.01.01-00-133/09-00, National Center of Science (NCN, Poland) as a research project in years 2011-2014 and project No. DEC-2011/03/N/ST3/02353 for years 2012-2014 and DFG in Germany.

\section{References}

[1] G.D. Mahan, Many-Particle Physics, 3rd ed., Kluwer, New York 2000.

[2] A.A. Abrikosov, L.P. Gorkov, I.E. Dzyaloshynski, Methods of Quantum Field Theory in Statistical Physics, Dover, New York 1963.

[3] A. Crépieux, P. Bruno, Phys. Rev. B 64, 014416 (2001).

[4] L.V. Keldysh, Zh. Eksp. Teor. Fiz. 47, 1515 (1964) [Sov. Phys. JETP 20, 1018 (1964)].

[5] E.M. Lifshitz, L.P. Pitaevskii, Physical Kinetics, Nauka, Moscow 1979.

[6] S. Onoda, N. Sugimoto, N. Nagaosa, Prog. Theor. Phys. 116, 61 (2006).

[7] V.K. Dugaev, P. Bruno, M. Taillefumier, B. Canals, C. Lacroix, Phys. Rev. B 71, 224423 (2005).

[8] J. Inoue, T. Kato, Y. Ishikawa, H. Itoh, G.E.W. Bauer, L.W. Molenkamp, Phys. Rev. Lett. 97, 046604 (2006).

[9] N.A. Sinitsyn, A.H. MacDonald, T. Jungwirth, V.K. Dugaev, J. Sinova, Phys. Rev. B 75, 045315 (2007); J. Sinova, D. Culcer, Q. Niu, N.A. Sinitsyn, T. Jungwirth, A.H. MacDonald, Phys. Rev. Lett. 92, 126603 (2004).

[10] J. Rammer, H. Smith, Rev. Mod. Phys. 58, 323 (1986).

[11] R. Raimondi, C. Gorini, P. Schwab, M. Dzierzawa, Phys. Rev. B 74, 035340 (2006).

[12] E.G. Mishchenko, B.I. Halperin, Phys. Rev. B 68, 045317 (2003); E.G. Mishchenko, A.V. Shytov, B.I. Halperin, Phys. Rev. Lett. 93, 226602 (2004); A.V. Shytov, E.G. Mishchenko, H.A. Engel, B.I. Halperin, Phys. Rev. Lett. 73, 075316 (2006).

[13] A. Khaetskii, Phys. Rev. Lett. 96, 056602 (2006); Phys. Rev. B 73, 115323 (2006).

[14] O.V. Dimitrova, Phys. Rev. B 71, 245327 (2005). 